\title{
Momba'etéva
}

(Lo que es significativo, relevante)

Revista de Investigaciones en Ciencias Jurídicas, Sociales y Políticas

\section{LA TEORÍA LIBERTARIA DE JUSTICIA DE ROBERT NOZICK. REFLEXIONES A PARTIR DEL PRINCIPIO DE ADQUISICIÓN INICIAL JUSTA}

\author{
ROBERT NOZICK'S LIBERTARIAN THEORY OF JUSTICE. \\ REFLECTIONS FROM PRINCIPLE OF JUSTICE IN ACQUISITION
}

\section{VICTORIA GUERRIERI}

Doctorando en Derecho, Facultad de Derecho Universidad Nacional del Nordeste. Becaria Doctoral UNNE-CONICET. Especialista en Derecho Tributario, Facultad de Derecho de la Universidad Nacional de Córdoba. Abogada, Facultad de Derecho de la UNNE. Profesora Jefe de Trabajos Prácticos de la Cátedra B de Filosofía del Derecho de la Facultad de Derecho de la UNNE. Integrante de Equipo Consolidado I+D en Estudios Críticos, Pluralismo Jurídico y Minorías Culturales de la UNNE.

Correo electrónico: vickyguerrieri@gmail.com

Pertenencia Institucional: Facultad de Derecho, Ciencias Sociales y Políticas de la Universidad Nacional del Nordeste. 


\section{RESUMEN}

El presente trabajo estudia la teoría libertaria de justicia de Robert Nozick a partir del análisis del "principio de adquisición inicial justa" postulado en dicha teoría y su relación con sus definiciones sobre el Estado Mínimo. El análisis advierte que se producen tensiones y contradicciones que parecieran ser insalvables.

Palabras clave: Justicia, Estado, Igualdad

\section{Abstract}

This paper studies Robert Nozick's libertarian theory of justice from the analysis of the "principle of justice in acquisition" postulated in his theory, and its relation with his definitions of the Minimum State. The analysis warns that there are tensions and contradictions that seem to be insurmountable.

Keywords: Justice, State, Equality

RECEPCIÓN: 3/10/2019

ACEPTACIÓN: 21/05/2020

\section{INTRODUCCIÓN}

El presente trabajo se propone analizar la teoría libertaria de justicia de Robert Nozick ${ }^{1}$, a partir de una objeción que se propone y que puede postularse de la siguiente manera: la falta de explicitación y desarrollo por parte de Robert Nozick del "principio de adquisición inicial justa”, postulado en su teoría de los derechos de propiedad, conlleva contradicciones que colisionan con sus definiciones sobre el Estado Mínimo.

Antes de desarrollar las razones en que se funda esta objeción, se hará una breve referencia a los aspectos más salientes de teoría de Robert Nozick.

Luego se abordará el análisis de las contradicciones que, en mi opinión, surgen del postulado propuesto; y finalmente, se exponen algunas conclusiones.

1. Robert Nozick (1938 - 2002), profesor en la Universidad de Harvard, conocido principalmente por su obra Anarquía, Estado y Utopía, donde critica la teoría distributiva y propone el Estado Mínimo como la forma de gobierno más justa. 


\section{NoZICK, TÍTULOS Y ESTADO MÍNIMO}

En su obra Anarquía, Estado y Utopía, Robert Nozick se dedica en gran parte a refutar a los argumentos planteados por John Rawls (1971) en su teoría sobre la justicia distributiva (Teoría sobre la Justicia).

Nozick (1988) parte de afirmar que si el mundo fuera completamente justo, tendrían que cumplirse ciertas definiciones inductivas de justicia sobre pertenencias a las que denomina "posesiones" de las personas.

A partir de allí, elabora una teoría de la justicia como derechos o, como lo denomina el autor, la justicia como "títulos".

Nozick selecciona un conjunto de derechos individuales: el derecho a la propiedad, a la vida y a la libertad. Considera a estos derechos como naturales, anteriores a cualquier sistema social y/o político, y los postula como la base de cualquier organización social.

Estos derechos son "títulos" que deben permanecer inalterables, sin que se les aplique ningún tipo de coerción que pueda violarlos.

La justicia en la teoría de Nozick se trata de los títulos o "tenencias" o posesiones, es decir, la justicia gira en torno a la propiedad. En este sentido, se podría decir que la justicia implica para Nozick que cada persona en ejercicio de sus derechos debe acceder a las posesiones sobre las que tiene un título.

Además, estos derechos se relacionan con las ideas de autonomía e independencia del individuo en el sentido de que cada persona es libre de elegir su forma de proceder, en tanto y en cuanto no vulnere derechos de otros.

Para Nozick (1988) una teoría de los derechos de propiedad necesita explicar tres principios diferentes:
(a) justicia en la apropiación inicial,
(b) justicia en la transferencia a partir de la apropiación inicial,
(c) justicia en la rectificación de violaciones a los principios de justicia que rigen la apropiación inicial y las transferencias que resultan de dicha apropiación.

Kymlicka (1995) describe a estos tres principios de la siguiente manera:

El principio de transferencia: cualquier cosa que sea justamente adquirida puede ser libremente transferida; 
El principio en la adquisición inicial justa: una explicación acerca del modo en que las personas, inicialmente, llegaron a poseer aquello que puede ser transmitido;

El principio de rectificación de la injusticia: cómo actuar frente a lo poseído si ello fuera injustamente adquirido o transferido. (p. 111)

Se podría decir entonces que para Nozick, la justicia se mide en la apropiación de posesiones, en su transferencia y la rectificación de adquisiciones y transferencias que hayan sido injustas.

Citando al propio Nozick, se ejemplifican cómo juegan estos principios:

Si soy dueño de una parcela de tierra, entonces el primer principio establece que soy libre de realizar cualquier transacción que desee con mi tierra. El segundo principio nos dice cómo comenzó la tierra a ser poseída. El tercer principio nos dice qué hacer en el caso que el primer o segundo principio resulten vulnerados. En su conjunto, implican que si las actuales pertenencias de la gente fueron justamente adquiridas, entonces la fórmula para una distribución justa es la de que "a cada uno como escoja, a cada uno según ha escogido" (citado en Kymlicka, 1995, p.112)

Por otro lado, es importante ver que Nozick (1988) refiere a que sólo un Estado Mínimo podría haber surgido sin violar los principios de justicia en la adquisición y transferencia de los derechos naturales a la propiedad del cuerpo, la vida y la libertad. Y en este sentido, Nozick rechaza cualquier intento de justificación de un Estado más extenso, por considerar que viola los derechos de las personas.

Es de destacar que al poner el énfasis en los derechos individuales, y en una opción por el Estado Mínimo, Nozick asume una posición libertaria sobre la justicia.

La vinculación que hacen los libertaristas entre la justicia y el mercado podría resumirse, conforme la teoría de Nozick, en la siguiente afirmación "cualquier distribución que resulte de transferencias libres a partir de una situación justa es en sí misma justa" Kymlicka, 1995, p. 111)

Kymlicka (1995) señala que los libertaristas defienden las libertades de mercado, y exigen la limitación del papel del Estado en cuanto a las políticas sociales. ${ }^{2}$

2. Resulta oportuno mencionar que si bien la obra de Nozick es considerada un clásico de los años setenta, muy en sintonía con la política de la nueva derecha de Margaret Thatcher y Ronald Reagan, tal como lo hace notar Kymlicka (1995) se deben tener presentes las diferencias entre un libertario y un neoconservador. Explica Brittan (1988) que ambos formaban parte de un movimiento a favor de las medidas políticas en defensa del libre mercado etiquetados como la "nueva derecha", pero los libertaristas defienden su compromiso con el mercado recurriendo a una noción más amplia de libertad personal (apoyaban la liberalización de las leyes relativas a la homosexualidad, el divorcio, el aborto etc.) viendo en ello una prolongación de su defensa del mercado. Por su parte, los neoconservadores se preocupan por reestablecer los valores tradicionales, todo lo cual puede implicar la limitación de "estilo de vida desaprobados" (citado en Kymlica, 1995) 
Nozick en su posición libertaria ha dicho que "Los individuos tienen derechos, y hay cosas que ninguna persona o grupo puede hacerles (sin violar sus derechos). Esto derechos son tan firmes y de tan largo alcance que surge la cuestión de qué pueden hacer los Estados y sus funcionarios, si es que algo pueden hacer".

Respecto de las funciones del Estado, plantea Nozick solo "un Estado mínimo limitado a las estrictas funciones de protección contra la violencia, el robo y el fraude, de cumplimiento de contratos, etc. se justifica; que cualquier Estado más amplio violaría el derecho de las personas de no ser obligadas a hacer ciertas cosas y, por tanto, no se justifica. Por ello no hay educación pública, ni atención sanitaria estatal ni cuidado del transporte, caminos o parques. Todas esas actividades implican una tributación coercitiva sobre cierta gente en contra de su voluntad, lo que vulnera el principio de que "a cada uno como escoja a cada uno según ha escogido".

Delineados los aspectos más salientes sobre las funciones del Estado Mínimo en la teoría de Nozick, cabe mencionar que el autor entiende al sistema impositivo como un sistema de trabajo forzado.

$\mathrm{Al}$ respecto ha expresado que "tomar las ganancias de $\mathrm{n}$ horas laborales es como tomar $\mathrm{n}$ horas de la persona; es como forzar a la persona trabajar $\mathrm{n}$ horas para propósitos de otra. (Nozick, 1988, p. 170).

Nozick (1988) considera al sistema impositivo como ilegítimo y para reflexionar al respecto se hace las siguientes preguntas:

¿Cómo puede ser legítimo para un sistema impositivo apoderarse de algunos de los bienes del hombre? ¿por qué debemos tratar al hombre cuya felicidad requiere de ciertos bienes o servicios materiales, de manera diferente de como tratamos al hombre cuyas preferencias y deseos hacen innecesarios tales bienes para su felicidad? ¿por qué el hombre que prefiere ver una película (y que tiene que ganar el dinero para pagar el boleto) debe estar expuesto al requerimiento de ayuda al necesitado, mientras que una persona que prefiere observar una puesta de Sol (y, por tanto, no necesita ganar dinero extra) no lo está? ¡No es, de hecho, sorprendente que los redistribucionistas decidan desentenderse del hombre cuyos placeres son tan fácilmente alcanzables sin trabajo extra, mientras que añaden, en cambio, una carga más al pobre infortunado que debe trabajar por sus placeres? (p.162)

Los ejemplos de Nozick resultan extremos y reflejan una postura a favor de la supresión de dichos sistemas.

Ya adentrándonos en el análisis sobre la teoría de Nozick, a primera vista, se advierte que en cada uno de los puntos, su teoría sobre de los derechos de propiedad y su relación con el Estado, aparece como fundada, coherente y justificada. 
Independientemente de si coincidimos o no por nuestras valoraciones subjetivas con la justicia o injusticia que deriva de guiarnos por los principios que propone, su construcción teórica resulta plausible.

Sin embargo, el presente trabajo se propone señalar algunos puntos en los cuales la teoría de Nozick aparece débil o deja vetas.

El principal problema que se encuentra es que Nozick no explica, ni fundamenta cómo se determina nada más ni nada menos que el punto de partida de su elaboración teórica: Nozick no se refiere de manera acabada a la "distribución inicial" y "apropiación inicial".

Por definición propone un punto de distribución inicial, que postula como justo, pero no desarrolla este punto. Deja en manos de los igualitaristas la distribución inicial, concediéndoles, a su elección, la forma de distribución que consideren.

Nozick es hábil al poner en cabeza de sus "contrincantes" este punto inicial, y sustraerse de dar una explicación.

Siendo que sobre este punto inicial luego se estructura toda su teoría, pareciera quedar inconclusa e inacabada su teorización sobre la distribución inicial, pues habla de "justicia en la apropiación inicial", justicia que implica que "hay algunos derechos de propiedad incuestionables distribuidos”, sin más.

Sea cual sea la posición que tomemos como punto de partida, ¿podemos considerarla equitativa y justa? ¿podemos considerar a esta “distribución inicial”, sin siquiera discutirla, como distributiva de un conjunto de derechos incuestionables sobre objetos particulares?

Es hábil Nozick, porque nos obliga a entrar en contradicción, pues siendo que, dada su propuesta, hemos "concedido" a individuos derechos incuestionables sobre objetos de manera incuestionable, claramente no podemos luego exigir un tributo sobre esa concesión que hemos decidido como incuestionable.

Como reflexiona Kymlicka (1995) tal vez, la mejor manera de responder a Nozick en este punto hubiera sido rehusarnos a definir cualquier distribución, más aún cuando su propuesta incluye el otorgamiento de derechos incuestionables, porque ya este punto de partida implica una distribución inicial que no es equitativa.

No obstante ello, Nozick avanza en su teoría a partir de este punto y, a través del análisis del ejemplo del basquetbolista Wilt Chamberlain, se harán cuestionamientos mediante los cuales se pretenden demostrar debilidades en la teoría de Nozick sobre la “distribución inicial” por presentar contradicciones con la idea de Estado Mínimo. 


\section{Contradicciones a PARTIR del análisis DEl EJemplo de Wilt Chamberlain}

Nozick (1988) intenta demostrar la "justicia en la transferencia”, es decir que cualquier cosa que sea justamente adquirida puede ser libremente transferida, a partir del ejemplo de personas que voluntariamente entregan sumas de dinero al basquetbolista Wilt Chamberlain.

Brevemente es necesario reseñar que el ejemplo de Nozick relata que Wilt Chamberlain, deportista muy solicitado por equipos de baloncesto por ser una gran atracción de taquilla, firma un contrato con un equipo por el cual, en cada partido jugado en casa, 25 centavos del precio de cada entrada serán para él, sin que ello implique un "saqueo a los propietarios".

Señala Nozick:

La temporada comienza y la gente alegremente asiste a los partidos de su equipo: las personas compran sus entradas, depositando veinticinco centavos del precio en una caja especial que tiene el nombre de Wilt Chamberlain (...) Supongamos que un millón de personas asisten a los partidos, y Wilt Chamberlain termina con 250.000 dólares, ingresos medios e incluso mayores que los de ningún otro (...) (p.163-164)

Tanto en el análisis posterior del ejemplo, como en el plano teórico, Nozick parte de que el Sr. Chamberlain tiene "títulos", o derechos de propiedad que serían incuestionables.

Para comenzar el análisis, se considera es necesario hacer diversas preguntas sobre esta “distribución inicial”. Por ejemplo, podríamos preguntar cómo llegó Wilt Chamberlain a ser jugador de basket, si fue apoyado por alguna institución, si recibió alguna beca universitaria, o si sólo y tan sólo llegó a ser una gran estrella por sus aptitudes físicas y esfuerzo personal. Podríamos indagar en cómo llegó a ser jugador de grandes equipos, cómo se financian esos equipos, qué influencia tiene la propaganda sobre los aficionados del basket como para que voluntariamente decidan entregar sumas a una estrella, etcétera.

Se hará referencia a algunas una de estas preguntas y seguramente surgirán otras, en relación con la teoría de Nozick.

Podríamos decir que a Wilt Chamberlain le fueron dadas oportunidades, ya sea por parte del Estado o por iniciativas privadas. ${ }^{3}$

Ahora, si se analiza la cuestión desde el punto de vista del Estado Mínimo, Nozick rechazaría, primero que la Universidad de Kansas, a la que asistió Chamberlain, cuente

3. Cabría preguntamos cuántos niños y jóvenes con grandes aptitudes no tienen estas mismas oportunidades. 
con financiamiento del Estado. Luego, se opondría a que la Universidad decidiera becar en su matrícula a Wilt Chamberlain.

Es decir, Nozick hubiera considerado que la carrera Chamberlain que le ha permitido la obtención de sus "títulos" es injusta, pues ha sido posible en virtud de un sistema que parte de una tributación coercitiva, pues la Universidad ha sido sustentada por personas que han sido obligadas al pago de tributos, en tanto el pago de un tributo no es voluntario. Para Nozick serían injustos estos títulos dado que las personas tienen derecho a sus posesiones, sólo sin fueron justamente adquiridas, dónde "derecho" significa "tener un derecho incuestionable para disponer libremente del modo en que uno lo considere conveniente, en tanto ello no implique el uso de la fuerza..."

Entonces, si bien Nozick deja librada la "distribución inicial”, ello representa un vacío en su teoría. Más aún, cuando vemos que el propio ejemplo que él mismo propone para el análisis, presenta antecedentes que se contradicen con su teoría.

Planteaba Nozick (1988) que sólo un Estado Mínimo podría haber surgido sin violar derechos individuales, en el sentido que él considera a los derechos, libres de toda injerencia estatal.

Dado lo expuesto, resulta contradictorio que Nozick pueda aceptar, sin más que los "títulos" o posesiones que fue adquiriendo Chamberlain tengan como antecedente el aporte contributivo de la sociedad.

Como se viene diciendo, si bien Nozick acepta esta "distribución inicial”, cualquiera que sea, del análisis en conjunto de los antecedentes y su intención de justificar las "transferencias”, surgen contradicciones.

Se podría decir que Wilt Chamberlain obtuvo sus "títulos", no sólo por sus "apropiaciones y transferencias justas" conforme la teoría de los derechos como títulos de Nozick; sino que Wilt Chamberlain tuvo la oportunidad -como no la tienen otros- de que su talento se viera acompañado por “injerencias” económicas y sociales, estatales y de particulares, que le han permitido adquirir esos "títulos". ${ }^{4}$

Entonces si bien Nozick intenta justificar el hecho de que Wilt Chamberlain tenga tantos más bienes que otra gente y tal vez más de lo que merece o necesita, y que ello es

4. Nos parece interesante adicionar como comentario que el personaje del ejemplo se consagró NBA, que es la liga nacional privada de baloncesto en los Estados Unidos. Cabe mencionar que hoy la propia NBA, que es una asociación de franquicias privadas, tiene un sistema de límite salarial. Este sistema se establece para evitar los equipos (franquicias) con mayores recursos económicos puedan obtener los mejores jugadores, y que aquellos equipos en desventaja económica, luego de un mal torneo puedan llegar a la quiebra. Nozick podría considerar injusto este límite existente en la NBA, pues podría sostener que cada franquicia, conforme su capacidad económica tiene la libertad de contratar y pagar lo que considere a cualquier jugador, sin restricción alguna. 
irrelevante para evaluar la justicia de esta situación, la circunstancia de que no explicite la justicia en la apropiación inicial hace tambalear tu teoría, si relacionamos este punto con sus teorizaciones sobre el Estado Mínimo.

Se reitera que para Nozick (1988) "cualquier Estado más extenso viola los derechos de las personas"(p.153), razón por la cual no podría Nozick justificar que Chamberlain tiene posesiones o títulos adquiridos de manera incuestionable.

Así, con un mínimo análisis de los antecedentes del caso, se ha intentado demostrar cómo asumir la postura sobre el Estado Mínimo impediría a Nozick justificar los títulos de Chamberlain.

\section{Conclusiones}

Se ha intentado demostrar, con el propio ejemplo que da el autor Nozick del basquetbolista Wilt Chamberlain, que su teoría presenta un vacío inicial, pues parte de que la situación inicial de posesiones Chamberlain sería consecuencia de una "distribución justa”, que al ser analizada, contradice sus postulados sobre el Estado Mínimo.

Como se ha dicho, si bien Nozick en todo momento deja en claro que no es él quien decide esta distribución inicial, esta postura resulta ser una opción cómoda para el autor, siendo que a partir de ello luego elabora su construcción teórica.

Se ha visto cómo de los antecedentes del caso, surgen contradicciones que resultan insalvables en lo que respecta a sus propias convicciones y teorizaciones.

La teoría de Nozick, a mi parecer, termina por justificar la desigualdad que presentan las sociedades. Podría decir además que es ajustada a la realidad, y hasta que resulta cómoda para ciertas corrientes de pensamiento conservadoras, pues permite describir -y justificar- las desigualdades, pretendiendo demostrar que no existe nada malo ni injusto en las mismas.

Finalmente se podría afirmar que la teoría de Nozick es más una teoría sobre la propiedad y la libertad, que sobre la justicia. 


\section{Bibliografía}

Kymlicka,W.(1995). Filosofía Politica Contemporánea. (Traducción de Roberto Gargarella) Barcelona: Ed. Ariel S.A.

Nozick, R. (1988). Anarquía, Estado y Utopía. Buenos Aires-México- Madrid: Ed. Claves. Fondo de Cultura Económica.

Rawls, J. (1971). Teoría de la Justicia. (Traducción González, M.D 6º impresión 2006) Cambridge: Harvard University Press. 